\title{
Laplace transform method for logistic growth in a population and predator models
}

\author{
Serdal Pamuk and Nagihan Soylu \\ Department of Mathematics, University of Kocaeli, Umuttepe Campus, Kocaeli, Turkey
}

Received: 25 November 2020, Accepted: 27 December 2020

Published online: 29 December 2020.

\begin{abstract}
We apply the Laplace transform method to logistic growth model in a population and prey-predator models.Among the techniques appearing in research literature, this method is very effective in terms of accuracy, stability and convergence of solutions. Analytical and numerical results are given and compared. We show that the Laplace transform method we have presented in this work gives accurate approximate solutions to nonlinear problems of physics, mathematics and biology.
\end{abstract}

Keywords: Laplace transform method, single species, interacting species, lotka-volterra systems

\section{Introduction}

Many scientists have tried to solve nonlinear differential equations appearing in the research literature [1,5,6,10-12]. However, most of the methods they use require a tedious analysis to solve their model equations. The Laplace transform method (LTM) is a particular integral transform invented by the French mathematician Pierre-Simon Laplace, and systematically developed by the British physicist Oliver Heaviside, to simplify the solutions of many differential equations that describe physical processes. LTM turns differential equations into polynomial equations, which are easier to handle. Once solved, use of the inverse Laplace transform method reverts to the time domain. However, for the nonlinear equations, LTM can not be used alone. Therefore, for the nonlinear case we use the series expansion of the dependent variable to get the solution.

In this paper we aim to give an application of this method to couple of nonlinear biological models that consist of a logistic growth model in a population and a prey-predator model. First, we study the logistic growth model in a population [9]

$$
\frac{d U}{d t}=s U(1-U / A)
$$

where $s$ and $A$ are positive constants. Here $U=U(t)$ represents the population of the species at time $t$, and $s U(1-U / A)$ is the per capita growth rate, and $A$ is the carrying capacity of the environment. Non-dimensionalization of Eq.(1) by setting [9]

$$
u(\tau)=\frac{U(t)}{A}, \quad \tau=s t
$$

\footnotetext{
*Corresponding author e-mail: spamuk@kocaeli.edu.tr
} 
results in

$$
\frac{d u}{d \tau}=u(1-u)
$$

If $U(0)=U_{0}$, then $u(0)=U_{0} / A$, and the analytical solution of Eq.(2) follows easily

$$
u(\tau)=\frac{1}{1+\left(A / U_{0}-1\right) e^{-\tau}} .
$$

Next, we study the Predator-Prey Models: Lotka-Volterra systems as an interacting species model defined by [9]

$$
\begin{aligned}
& \frac{d U}{d \tau}=U(e-V f), \\
& \frac{d V}{d \tau}=V(g U-h),
\end{aligned}
$$

where $e, f, g$ and $h$ are constants. Here $U=U(t)$ is the prey population and $V=V(t)$ that of the predator at time $t$. We can non-dimensionalize the system (4)-(5) [9] by setting

$$
u(\tau)=\frac{g U(t)}{h}, v(\tau)=\frac{f V(t)}{e}, \tau=e t, \beta=h / e,
$$

and it becomes

$$
\begin{aligned}
& \frac{d u}{d \tau}=u(1-v), \\
& \frac{d v}{d \tau}=\beta[g(u, v)-v] .
\end{aligned}
$$

In section 2, we apply the LTM $[2,3,7,8]$ to the models of the form Eq.(2) and Eqs.(6)-(7), respectively.

\section{Laplace transform method (LTM)}

\subsection{LTM for logistic growth model}

In this subsection we consider the model equation of the form

$$
\frac{d u}{d \tau}=u-f(u), \quad u(0)=u_{0},
$$

where $f$ is a nonlinear function of $u$. We look for the solution $u$ satisfying (8). Therefore, we assume the solution, $u$ of (8) has an infinite series representation of the form

$$
u=u(\tau)=\sum_{n=0}^{\infty} a_{n} \tau^{n}
$$

and it satisfies the required conditions for the existence of the Laplace transform, $\mathscr{L}$ defined by

$$
\mathscr{L}(u(t))=\int_{0}^{\infty} e^{-s t} u(t) d t, t \geq 0,
$$


provided that this integral exists. Applying the Laplace transform to the both sides of the differential equation in (8) we get

$$
s U(s)-u_{0}=U(s)-F(s),
$$

where $U(s)=\mathscr{L}(u(t))$ and $F(s)=\mathscr{L}\{f(u(t))\}$ are the Laplace transforms of the functions $u(t)$ and $f(u(t))$, respectively. Solving (11) for $U(s)$ we obtain

$$
U(s)=u_{0} \frac{1}{s-1}-\frac{F(s)}{s-1} .
$$

Therefore, assuming the inverse Laplace transform $\mathscr{L}^{-1}$ exists and applying it to (12) we end up with

$$
u(t)=u_{0} \exp (t)-\mathscr{L}^{-1}\left(\frac{F(s)}{s-1}\right) .
$$

\subsection{LTM for prey-predator model}

In this subsection we study the system of non-linear differential equations of the form

$$
\begin{aligned}
& \frac{d u}{d \tau}=u-f(u, v), \\
& \frac{d v}{d \tau}=\beta[g(u, v)-v],
\end{aligned}
$$

with initial conditions

$$
u(0)=u_{0}, \quad v(0)=v_{0},
$$

where $f$ and $g$ are nonlinear functions of $u$ and $v$. Here $\beta$ is some positive constant. We look for the solutions $(u, v)$ satisfying (14)-(16). We assume the solutions $u$ and $v$ of the system (14)-(15) have the following infinite series expansions of the form

$$
u=u(\tau)=\sum_{n=0}^{\infty} a_{n} \tau^{n}, \quad v=v(\tau)=\sum_{n=0}^{\infty} b_{n} \tau^{n},
$$

and they satisfy the required conditions for the existence of their Laplace transforms. Applying the Laplace transform to the system (14)-(15) and using (16) we get

$$
\begin{aligned}
& s U(s)-u_{0}=U(s)-F(s), \\
& s V(s)-v_{0}=\beta(G(s)-V(s)),
\end{aligned}
$$

where $U(s)=\mathscr{L}(u(t)), F(s)=\mathscr{L}\{f(u(t), v(t))\}, V(s)=\mathscr{L}(v(t)), G(s)=\mathscr{L}\{g(u(t), v(t))\}$ are the Laplace transforms of the functions $u(t), f(u(t), v(t)), v(t)$ and $g(u(t), v(t))$, respectively. 
Solving (18) for $U(s)$ and $V(s)$ we get

$$
\begin{aligned}
& U(s)=u_{0} \frac{1}{s-1}-\frac{F(s)}{s-1} \\
& V(s)=v_{0} \frac{1}{s+\beta}+\beta \frac{G(s)}{s+\beta}
\end{aligned}
$$

If we assume the inverse Laplace transforms exist and apply them to the system (19) we find

$$
\begin{aligned}
& u(t)=u_{0} \exp (t)-\mathscr{L}^{-1}\left(\frac{F(s)}{s-1}\right), \\
& v(t)=v_{0} \exp (-\beta t)+\beta \mathscr{L}^{-1}\left(\frac{G(s)}{s+\beta}\right),
\end{aligned}
$$

which are the desired solutions of the initial value problem (14)-(16).

\section{Applications and results}

Example 1. We consider the initial value problem (8). For numerical computations we take $N_{0}=2$ and $A=1$. Therefore, $u_{0}=N_{0} / A=2$. We let $f(u)=u^{2}$ as in (2) so that one has

$$
f(u)=\left(\sum_{n=0}^{\infty} a_{n} t^{n}\right)^{2}=a_{0}^{2}+2 a_{0} a_{1} t+\left(2 a_{0} a_{2}+a_{1}^{2}\right) t^{2}+\left(2 a_{0} a_{3}+2 a_{1} a_{2}\right) t^{3}+\cdots
$$

We then obtain

$$
F(s)=\frac{a_{0}^{2}}{s}+\frac{2 a_{0} a_{1}}{s^{2}}+\frac{4 a_{0} a_{2}+2 a_{1}^{2}}{s^{3}}+\frac{12 a_{0} a_{3}+12 a_{1} a_{2}}{s^{4}}+\cdots
$$

Using (12) one gets

$$
\begin{aligned}
U(s) & =\frac{2}{s-1}-\frac{F(s)}{s-1} \\
& =\frac{2}{s-1}-\left\{\frac{a_{0}^{2}}{s(s-1)}+\frac{2 a_{0} a_{1}}{s^{2}(s-1)}+\frac{4 a_{0} a_{2}+2 a_{1}^{2}}{s^{3}(s-1)}+\frac{12 a_{0} a_{3}+12 a_{1} a_{2}}{s^{4}(s-1)}+\cdots\right\} .
\end{aligned}
$$

If we apply the inverse Laplace transform to this equation and use (9) we get

$$
\begin{aligned}
a_{0}+a_{1} t+a_{2} t^{2}+a_{3} t^{3}+\ldots= & 2\left(1+t+\frac{t^{2}}{2 !}+\frac{t^{3}}{3 !}+\ldots\right) \\
& -a_{0}^{2} t-\left(\frac{a_{0}^{2}}{2}+a_{0} a_{1}\right) t^{2}-\left(\frac{a_{0}^{2}}{6}+\frac{a_{0} a_{1}}{3}+\frac{2 a_{0} a_{2}}{3}+\frac{a_{1}^{2}}{3}\right) t^{3}-\cdots \\
= & 2+\left(2-a_{0}^{2}\right) t+\left(1-\frac{a_{0}^{2}}{2}-a_{0} a_{1}\right) t^{2} \\
& +\left(\frac{1}{3}-\frac{a_{0}^{2}}{6}-\frac{a_{0} a_{1}}{3}-\frac{2 a_{0} a_{2}}{3}-\frac{a_{1}^{2}}{3}\right) t^{3}+\cdots
\end{aligned}
$$


Equating coefficients of power $t$ yields

$$
\begin{aligned}
& a_{0}=2, \quad a_{1}=2-a_{0}^{2} \Longrightarrow a_{1}=-2, \\
& a_{2}=1-\frac{a_{0}^{2}}{2}-a_{0} a_{1} \Longrightarrow a_{2}=3, \\
& a_{3}=\frac{1}{3}-\frac{a_{0}^{2}}{6}-\frac{a_{0} a_{1}}{3}-\frac{2 a_{0} a_{2}}{3}-\frac{a_{1}^{2}}{3} \Longrightarrow a_{3}=-\frac{13}{3},
\end{aligned}
$$

As a result, one obtains the solution $u(t)$ from (9) as follows

$$
u(t)=2-2 t+3 t^{2}-\frac{13}{3} t^{3}+\cdots,
$$

which is the exactly solution obtained in Eq.(3) in the closed form. This solution is precisely the same as the one we obtained in [4].

Let $\phi_{n}(t)$ be the $n$th partial sums of the series (9), i.e,

$$
\phi_{n}(t)=\sum_{k=0}^{n} a_{k} t^{k}
$$

As one sees from Fig.1 that we have obtained a very close approximation to the exact solution of logistic growth model in the time interval $[0,0.15]$ by calculating only 4 terms of the series in (23). This tells us that the speed of convergence of LTM is very fast. Of course, the total errors can be made small, for $t>0.15$ by finding new terms of the related series.

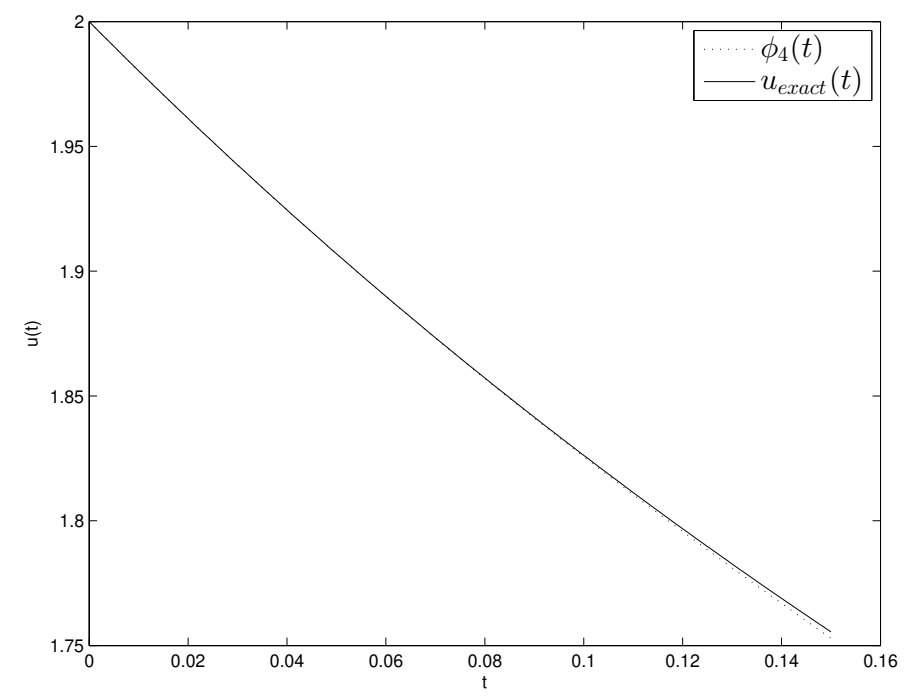

Fig. 1: Solution to the logistic growth model in a population. 
Example 2. We now solve the problem (14)-(16) with initial data $u(0)=1.3, v(0)=0.6$. We proceed as in section 2.2 . We take $\beta=1, f(u, v)=g(u, v)=u v$ in (14)-(15) so that we have

$$
\begin{aligned}
f(u, v)=g(u, v) & =\left(\sum_{n=0}^{\infty} a_{n} t^{n}\right)\left(\sum_{n=0}^{\infty} b_{n} t^{n}\right) \\
& =a_{0} b_{0}+\left(a_{0} b_{1}+a_{1} b_{0}\right) t+\left(a_{0} b_{2}+a_{1} b_{1}+a_{2} b_{0}\right) t^{2}+\cdots
\end{aligned}
$$

and the corresponding Laplace transforms of these functions become

$$
F(s)=G(s)=\frac{a_{0} b_{0}}{s}+\frac{a_{0} b_{1}+a_{1} b_{0}}{s^{2}}+\frac{2 a_{0} b_{2}+2 a_{1} b_{1}+2 a_{2} b_{0}}{s^{3}}+\cdots .
$$

Using (19) one gets

$$
\begin{aligned}
U(s) & =\frac{1.3}{s-1}-\frac{F(s)}{s-1}, \\
& =\frac{1.3}{s-1}-\left\{\frac{a_{0} b_{0}}{s(s-1)}+\frac{a_{0} b_{1}+a_{1} b_{0}}{s^{2}(s-1)}+\frac{2 a_{0} b_{2}+2 a_{1} b_{1}+2 a_{2} b_{0}}{s^{3}(s-1)}+\cdots\right\}, \\
V(s) & =\frac{0.6}{s+1}+\frac{G(s)}{s+1}, \\
& =\frac{0.6}{s+1}+\left\{\frac{a_{0} b_{0}}{s(s+1)}+\frac{a_{0} b_{1}+a_{1} b_{0}}{s^{2}(s+1)}+\frac{2 a_{0} b_{2}+2 a_{1} b_{1}+2 a_{2} b_{0}}{s^{3}(s+1)}+\cdots\right\} .
\end{aligned}
$$

If we apply the inverse Laplace transform to these equation and use (17) we get

$$
\begin{aligned}
a_{0}+a_{1} t+a_{2} t^{2}+a_{3} t^{3}+\ldots & =1.3\left(1+t+\frac{t^{2}}{2 !}+\frac{t^{3}}{3 !}+\cdots\right) \\
& -a_{0} b_{0} t-\left(a_{0} b_{0}+a_{0} b_{1}+a_{1} b_{0}\right) \frac{t^{2}}{2 !} \\
& -\left(a_{0} b_{0}+a_{0} b_{1}+a_{1} b_{0}+2 a_{0} b_{2}+2 a_{1} b_{1}+2 a_{2} b_{0}\right) \frac{t^{3}}{3 !}-\cdots \\
& =1.3+\left(1.3-a_{0} b_{0}\right) t+\left(1.3-a_{0} b_{0}-a_{0} b_{1}-a_{1} b_{0}\right) \frac{t^{2}}{2 !} \\
& +\left(1.3-a_{0} b_{0}-a_{0} b_{1}-a_{1} b_{0}-2 a_{0} b_{2}-2 a_{1} b_{1}-2 a_{2} b_{0}\right) \frac{t^{3}}{3 !}+\cdots,
\end{aligned}
$$

and

$$
\begin{aligned}
b_{0}+b_{1} t+b_{2} t^{2}+b_{3} t^{3}+\ldots & =0.6\left(1-t+\frac{t^{2}}{2 !}-\frac{t^{3}}{3 !}+\cdots\right)+a_{0} b_{0} t+\left(a_{0} b_{1}+a_{1} b_{0}-a_{0} b_{0}\right) \frac{t^{2}}{2 !} \\
& +\left(a_{0} b_{0}-a_{0} b_{1}-a_{1} b_{0}+2 a_{0} b_{2}+2 a_{1} b_{1}+2 a_{2} b_{0}\right) \frac{t^{3}}{3 !}-\cdots \\
& =0.6+\left(a_{0} b_{0}-0.6\right) t+\left(0.6-a_{0} b_{0}+a_{0} b_{1}+a_{1} b_{0}\right) \frac{t^{2}}{2 !} \\
& +\left(-0.6+a_{0} b_{0}-a_{0} b_{1}-a_{1} b_{0}+2 a_{0} b_{2}+2 a_{1} b_{1}+2 a_{2} b_{0}\right) \frac{t^{3}}{3 !}+\cdots
\end{aligned}
$$


Equating coefficients of power $t$ yields

$$
\begin{aligned}
& a_{0}=1.3, \quad a_{1}=1.3-a_{0} b_{0} \Longrightarrow a_{1}=0.52 \\
& a_{2}=\frac{1}{2 !}\left(1.3-a_{0} b_{0}-a_{0} b_{1}-a_{1} b_{0}\right) \Longrightarrow a_{2}=-0.013 \\
& a_{3}=\frac{1}{3 !}\left(1.3-a_{0} b_{0}-a_{0} b_{1}-a_{1} b_{0}-2 a_{0} b_{2}-2 a_{1} b_{1}-2 a_{2} b_{0}\right) \Longrightarrow a_{3}=-0.1122 \\
& \vdots \\
& b_{0}=0.6, \quad b_{1}=a_{0} b_{0}-0.6 \Longrightarrow b_{1}=0.18 \\
& b_{2}=\frac{1}{2 !}\left(0.6+a_{0} b_{1}+a_{1} b_{0}-a_{0} b_{0}\right) \Longrightarrow b_{2}=0.183 \\
& b_{3}=\frac{1}{3 !}\left(-0.6+a_{0} b_{0}-a_{0} b_{1}-a_{1} b_{0}+2 a_{0} b_{2}+2 a_{1} b_{1}+2 a_{2} b_{0}\right) \Longrightarrow b_{3}=0.0469
\end{aligned}
$$

The remaining terms of the series may be obtained in this manner. Writing these terms into Eq.(17) we get the approximate solutions to the problem (14)-(16):

$$
\begin{aligned}
& u(t)=1.3+0.52 t-0.013 t^{2}-0.1122 t^{3}-\cdots, \\
& v(t)=0.6+0.18 t+0.183 t^{2}+0.0469 t^{3}+\cdots,
\end{aligned}
$$

which are exactly the same as the ones we obtained in [4].

Figures (2) \& (3) show the comparisions of the numerical solutions and Laplace transform solutions of the system (14)-(16). Numerical solutions of the system are obtained with the built-in Matlab ordinary differential equation solver (Ode45) while Laplace transform solutions are obtained by using only four terms of the series.

It is seen from the last two figures that the numerical solutions and Laplace transform solutions of the system (14)-(16) are very close to each other in the time interval $[0,0.6]$. A further approximation may be achieved by calculating new terms to the Laplace transform solutions for $t \geq 0.6$.

As result, the Laplace transform method studied in this work gives very accurate approximate solutions to nonlinear problems of physics, mathematics, biology, etc. It also does not require linearization and biologically unrealistic assumptions.

\section{Competing interests}

The authors declare that they have no competing interests.

\section{Authors' contributions}

All authors have contributed to all parts of the article. All authors read and approved the final manuscript. 


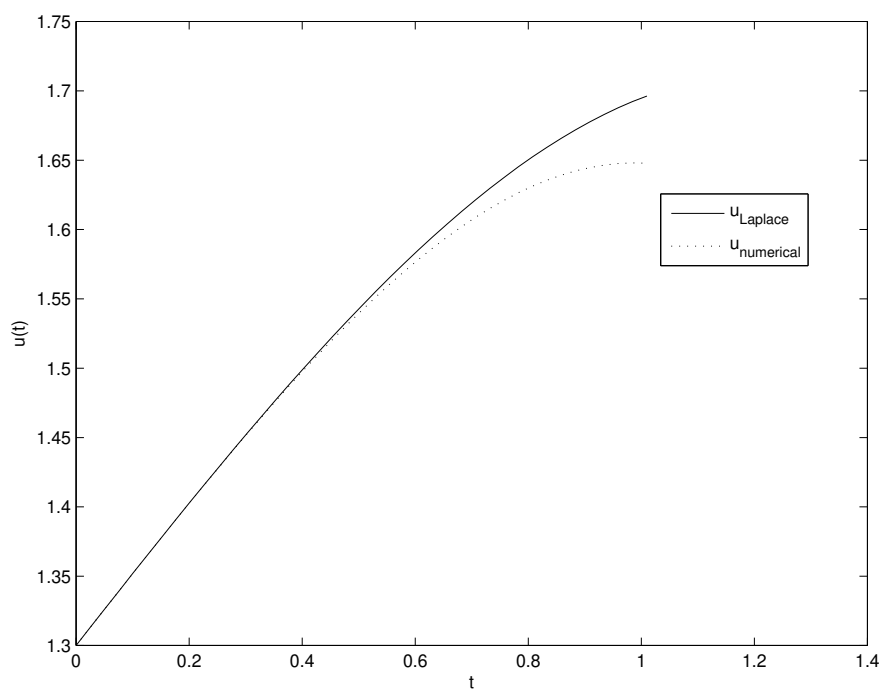

Fig. 2: Solutions to the system (14)-(16) showing the Prey Population.

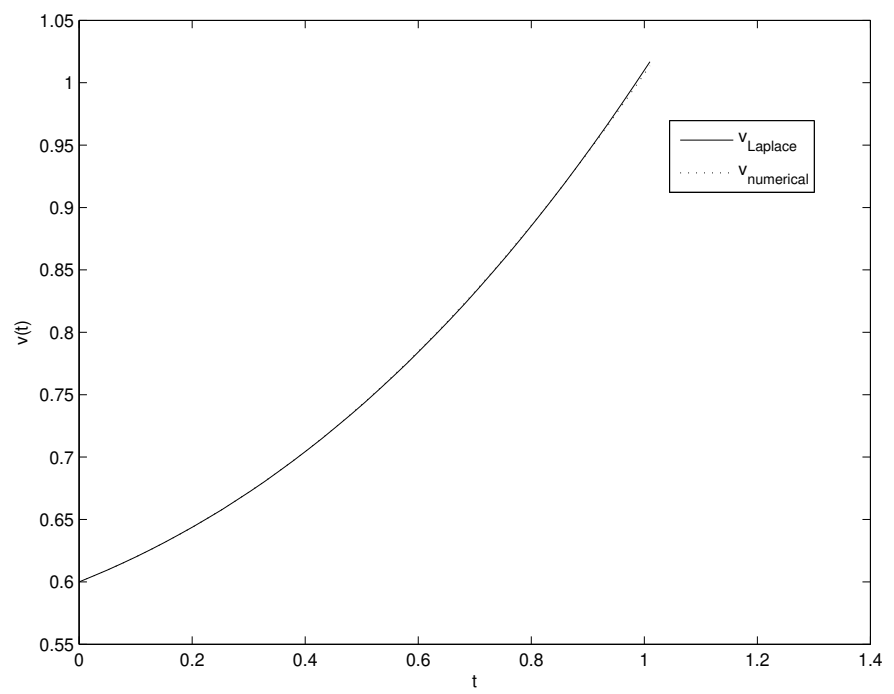

Fig. 3: Solutions to the system (14)-(16) showing the Predator Population.

\section{References}

[1] Adomian G., Rach R., 1986, On the Solution of Nonlinear Differential Equations with Convolution Product Nonlinearities, Journal of Mathematical Analysis And Applications, 114, pp. 171-175.

[2] Al-Saar F. M., Ghadle K. P., 2018, Combined Laplace Transform with Analytical Methods Solving Volterra Integral Equations with a Convolution Kernel, The Korean Jornal of Mathematics, 22, pp. 125-136.

[3] Handibag S., Karande B. D., 2012, Laplace Substitution Method For Solving Partial Differential Equations Involving Mixed Partial Derivatives, International Journal of Pure and Applied Mathematics, 78, pp. 973-979.

[4] Pamuk S., 2005, The decomposition method for continuous population models for single and interacting species, Applied Mathematics and Computation, 163, pp. 79-88. 
[5] Pamuk S., 2005, An application for linear and nonlinear heat equations by Adomian's decomposition method, Applied Mathematics and Computation, 163, pp. 89-96.

[6] Pamuk S., 2005, Solution of the porous media equation by Adomian's decomposition method, Physics Letters A, 344 , pp. 184-188.

[7] Kumari K., Gupta P. K., 2014, Application of Laplace-Differential Transform Method to Solve Nonlinear PDEs with Boundary Conditions, 3rd International Conference On "Innovative Approach in Applied Physical, Mathematical/Statistical, Chemical Sciences and Emerging Energy Technology for Sustainable Development”, New Delhi, Delhi, India.

[8] Davis H. T., 1962, Introduction to Nonlinear Differential and Integral Equations, New York:Dover.

[9] Murray J. D., 1993, Mathematical Biology, Springer, Berlin.

[10] Polyanin D. A., Zaitsevi F. V., 2004, Handbook of Nonlinear Partial Differential Equations, Chapman \& Hall /CRC, New York.

[11] Schiff J. L., 1999, The Laplace Transform: Theory and Applications, Springer, New York.

[12] Wazwaz A. M., 2011, Linear and Nonlinear Integral Equations, Springer, Berlin, Heidelberg. 